\title{
The natural history of unruptured intracranial aneurysms
}

\author{
J Mocco, M.D., Ricardo J. Komotar, M.D., Sean D. Lavine, M.D., \\ Philip M. Meyers, M.D., E. Sander Connolly, M.D., And Robert A. Solomon, M.D. \\ Department of Neurological Surgery, Columbia University, New York, New York
}

\begin{abstract}
Since the publication of preliminary results from the International Study of Unruptured Intracranial Aneurysms in 1998 there has been a great deal of debate concerning the natural history of these lesions and their attendant risk of aneurysmal subarachnoid hemorrhage. Therefore, the authors reviewed a selected number of crucial studies concerning this topic to determine the best evidence-based estimate of a rupture rate for these lesions. Based on this analysis, the yearly risk of bleeding for an unruptured intracranial aneurysm is estimated to be approximately $1 \%$ for aneurysms 7 to $10 \mathrm{~mm}$ in diameter. This risk of rupture increases with aneurysm size and it likewise diminishes as the size of the lesion decreases. This general rule serves as a reasonable interpretation of the results reported in the current body of literature.
\end{abstract}

\author{
KEY WORDS • aneurysm • natural history • risk of rupture • \\ subarachnoid hemorrhage • unruptured aneurysm
}

Over the past 5 years there has been a great deal of contention regarding the appropriate management of unruptured intracranial aneurysms. The kindling for this heated debate was the publication of preliminary results from the ISUIA $^{1}$ in the New England Journal of Medicine in December of 1998. Much of the discussion has centered on the natural history of unruptured aneurysms and the attendant risk of aneurysmal SAH. The ISUIA investigators made claims regarding the risk of aneurysmal SAH from small $(<10 \mathrm{~mm})$ aneurysms that were drastically lower (by a factor of 10-20) than what had been generally accepted previously. The result has been an enormous volume of impassioned editorials, balanced discussion, and new attempts at evidence-based assessment.

\section{PURPOSE}

For the general neurosurgeon, referring physician, or neurosurgeon in training, the evaluation of this literature can be both demanding and confusing, making the development of a sound evidence-based opinion on this matter a near impossibility. Therefore, we have critically reviewed a number of recent relevant studies to reach a set of conclusions concerning the natural history of unruptured intracranial aneurysms that are grounded in the best available data.

Abbreviations used in this paper: ICA = internal carotid artery; ISUIA = International Study of Unruptured Intracranial Aneurysms; $\mathrm{PCoA}=$ posterior communicating artery; $\mathrm{SAH}=$ subarachnoid hemorrhage.

\section{Relevant Study Designs}

The gold standard for evidence-based data is the randomized experimental trial. Unfortunately, given the devastating consequences and rare incidence of aneurysmal $\mathrm{SAH}$, as well as strong clinician preference regarding management of unruptured intracranial aneurysms, performing a randomized trial is essentially impossible. Interestingly, in some recent (and strongly debated) reports, investigators have intimated that a well-designed observational study can provide estimates that are highly consistent with those of randomized experimental trials., ${ }^{3,4}$ In any case, the observational study is currently the best source of data the medical community has for evaluating the natural history of unruptured aneurysms.

Unfortunately, observational studies often have serious flaws that must be taken into account. One such fundamental flaw is the bias involved in patient selection. By definition, an observational study consists of a population that has already undergone a selection process. Therefore, extreme care must be exercised when applying observational cohort data to the population at large, because the results are not derived from random sampling.

In addition to observational cohort studies, many investigators have used screening methods (that is, autopsy or angiography series) to establish the prevalence of unruptured intracranial aneurysms. Once both the prevalence of these lesions and the incidence of aneurysmal SAH in the population are known, simple calculations are used to determine the rate of rupture. Limitations include the quality of the screening technique and bias involved in evaluating patients who have been selected to undergo such screening processes. 
Regardless of the study design, the medical community must critically evaluate available data. It is only through meticulous effort that we may develop the best possible estimate of the natural history of unruptured aneurysms, and in the current age of evidence-based medicine both our colleagues and our patients deserve nothing less. To this end, we have reviewed a selected number of crucial studies concerning the natural history of unruptured intracranial aneurysms. These include two high-impact ISUIA papers; ${ }^{1,24}$ the study by Juvela, et al., ${ }^{10}$ in the Journal of Neurosurgery that included a $100 \%$ follow up over an 18 year period; the metaanalysis by Rinkel, et al., ${ }^{18}$ that includes the relevant literature published from 1955 to 1996; and the large single-institution angiographically assessed prevalence analysis performed by Winn, et al. ${ }^{25}$

\section{Selected Studies}

The ISUIA is an ongoing international collaboration of major neurosurgical centers organized to evaluate the natural history of unruptured intracranial aneurysms and the results of interventions used to treat these lesions. To date it has resulted in two seminal studies published over a 5year period. These studies have had a major impact on how this disease is evaluated and have stimulated much of the current debate surrounding treatment of unruptured aneurysms.

The first study, ${ }^{1}$ published in the New England Journal of Medicine, contained a retrospective component designed to assess the natural history of unruptured intracranial aneurysms. In this analysis the authors reviewed 1449 patients with 1937 aneurysms. These patients were divided into two groups: 727 who had no history of aneurysmal SAH (Group 1), and 722 who had a history of aneurysmal $\mathrm{SAH}$ from a different lesion (Group 2). The mean followup duration was 8.3 years. For Group 1 the rate of SAH for aneurysms smaller than $10 \mathrm{~mm}$ was $0.05 \%$ per year, whereas for aneurysms larger than $10 \mathrm{~mm}$ it was approximately $1 \%$ per year. For Group 2 the SAH rate was $0.5 \%$ for aneurysms smaller than $10 \mathrm{~mm}$ and approximately $1 \%$ per year for aneurysms larger than $10 \mathrm{~mm}$. These numbers were drastically lower than in the previous estimates. ${ }^{2,5,6}$, 9,12,13,15,18-20,22,23,26 Evaluations of predictors of rupture found that increasing size and location (posterior circulation and $\mathrm{PCoA}$ ) were significantly associated with rupture for Group 1, whereas location (basilar tip) and increasing age were predictive of rupture for Group 2.

Although the large number of aneurysms and the multicenter nature of this study certainly make it an important contribution to the literature, it has a number of serious design flaws. The cohort of patients that made up the retrospective evaluation of the natural history of unruptured intracranial aneurysms was subjected to a very significant selection bias. All patients had already been evaluated for surgery and selected for observation by the participating neurosurgeon. Clearly this is a very different population from that of all patients with unruptured aneurysms. Because these patients had already been selected for conservative management of their lesions, it is likely that they harbored extremely low-risk aneurysms due to location (cavernous carotid aneurysms were identified in Groups 1 and 2 at rates of 16.9 and $9.5 \%$, respectively) or small size (in Groups 1 and 2, 2- to 5-mm aneurysms were identified in 32.7 and $61.2 \%$ of patients, respectively). In addition, it is likely that the patients were medically ill, with increased deaths due to other causes removing them from the pool of possible patients with aneurysmal SAH. Last, patients whose lesions were originally selected for conservative management by their surgeons may have later crossed over to surgical treatment because of changes in symptoms or aneurysm size. This crossover would remove patients who were imminently at risk for $\mathrm{SAH}$, possibly lowering the observed rupture rate.

The second study, ${ }^{24}$ published in the journal Lancet, contains a prospective evaluation of the natural history of 2686 unruptured intracranial aneurysms in 1692 patients. In this analysis there were 1077 patients in Group 1 (those with no history of aneurysmal SAH), and 615 patients in Group 2 (those with a history of SAH). The mean followup duration was 4.1 years. Calculating the total risk of rupture for patients in both Groups 1 and 2, excluding those with aneurysms in the cavernous ICA, demonstrates that for aneurysms 7 to 12,13 to 24 , and greater than 25 $\mathrm{mm}$ in diameter the yearly rupture rates are $1.2,3.1$, and $8.6 \%$, respectively. Unfortunately, the results for aneurysms smaller than $7 \mathrm{~mm}$ were presented in a stratified manner and therefore cannot be effectively combined. For patients in Group 2, aneurysms smaller than $7 \mathrm{~mm}$, excluding those in the cavernous ICA, demonstrated a rupture rate of $0.4 \%$ per year, whereas for patients in Group 1 , lesions smaller than $7 \mathrm{~mm}$, excluding cavernous ICA aneurysms, demonstrated a $0.15 \%$ yearly rupture rate.

It is important to note that, as indicated in the ISUIA results published in the New England Journal of Medicine, there is certainly an unspecified selection bias in the population studied. Of even greater concern is the fact that, because this is a prospective study, we are also able to determine the proportion of patients who were excluded from the analysis, despite the intent to treat their lesions with conservative management. For instance, of the 1692 patients, 534 were switched to a therapeutic intervention (410 surgical, 124 endovascular) and were removed from follow up. It is not unreasonable to conclude that in at least some significant portion of these crossover patients the management strategy was changed because of either an increase in aneurysm size or the development of new symptoms, both of which have been implicated in large increases in rupture rate. Therefore, the population studied was not only created with an obvious selection bias, but also underwent a second selection process, by which aneurysms that may have been likely to rupture were removed from the cohort, despite the original intent to treat conservatively.

Also of note, 193 patients (11\%) died of causes other than aneurysmal SAH; these patients were removed from the analysis. This is a reasonable statistical technique, but it is troubling that 52 of these patients died of intracranial hemorrhage. It is unclear whether there was adequate evaluation to ensure that these intracranial hemorrhages were not due to aneurysms. Overall, it is exciting to see the neurosurgical community working to develop prospective natural history data pertaining to unruptured intracranial aneurysms, but we must exercise extreme caution when evaluating these studies and attempting to extrapolate them to the population at large. 
Juvela and colleagues ${ }^{10}$ provide a comprehensive observational cohort study that lacks the inherent bias of surgical selection found in the ISUIA. This is because they examined all patients with unruptured intracranial aneurysms seen at their institution over a given time period. This study was achievable because it was their department policy to manage all unruptured aneurysms conservatively prior to 1979 . In addition, the uniqueness of the sociomedical structure in Finland allowed $100 \%$ follow up to record outcome over a longer period of time (mean 18.1 years) than has been achieved in any other study. ${ }^{14,16}$

A total of 142 patients with 181 unruptured intracranial aneurysms were studied. The cumulative rate of aneurysmal SAH was $10.5 \%$ at 10 years, $23 \%$ at 20 years, and $30.3 \%$ at 30 years after the diagnoses were made. Aneurysm size and patient age (inversely) were significant predictors of aneurysmal SAH, as was active cigarette smoking $(\mathrm{p}<0.05$ for each). Major flaws in this study are the small total number of patients, the homogeneity of the study population (it is debatable whether the Finnish population's natural history can be extrapolated to other populations), and the overwhelming proportion (92\%) of patients with previous aneurysmal SAH (it is unclear whether the natural history of incidental aneurysms is the same as that of secondarily discovered lesions after aneurysmal SAH). Despite these major flaws, the lack of a surgical selection bias and the outstanding long-term follow up ensure this study's equal footing with the ISUIA as far as providing imperfect but valuable data regarding the estimate of bleeding rates for unruptured intracranial aneurysms.

Rinkel, et al., ${ }^{18}$ published an invaluable analysis of the natural history of unruptured intracranial aneurysms in which they performed a thorough review of the literature published between 1955 and 1996. To estimate the prevalence of unruptured aneurysms, data were summed from 23 studies (eight autopsy, 15 angiography) to evaluate 56,304 patients. The overall prevalence of these lesions in adults with no known specific risk factors (autosomal-dominant polycystic kidney disease, family history, ath-erosclerosis) was $2.3 \%$. The method of aneurysm detection strongly influenced the estimate of prevalence; retrospective autopsy, prospective autopsy, retrospective angiographic, and prospective angiographic studies demonstrated rates of $0.4,3.6,3.7$, and $6 \%$, respectively.

For analysis of the bleeding rate of unruptured intracranial aneurysms, the authors identified nine studies in which a total of 3907 patients were evaluated. The overall risk of rupture was $1.9 \%$ per year $(0.7 \%$ for unruptured aneurysms $\leq 10 \mathrm{~mm}$ and $4 \%$ for intact lesions $>10 \mathrm{~mm}$ ). It is important to realize that with any metaanalysis the final conclusions are only as good as the quality of the studies that are pooled to generate the data. That being said, the sheer number of patients analyzed and the detailed analysis performed by the authors make this an important investigation into the natural history of unruptured intracranial aneurysms. Additionally, the results reported by Rinkel, et al., are strengthened by the fact that their estimates of the prevalence of and incidence of bleeding in unruptured intracranial aneurysms corroborate with each other when calculated using the known incidence of aneurysmal SAH.
A final paper that has recently shed light on the natural history of unruptured intracranial aneurysms is one published by Winn, et al. ${ }^{25}$ In this paper, the authors reviewed the radiographic reports for all 4568 cerebral angiograms performed between April 1969 and January 1980 at the University of Virginia. Of the patients in whom these angiograms were obtained, 884 were excluded because of a history of SAH, symptomatic aneurysms, repeated angiography, solely cervical angiography, symptomatic arteriovenous malformations, and misregistration, leaving 3684 patients. The authors found a $0.65 \%$ prevalence of unruptured intracranial aneurysms.

It is important to note that this study had a number of possible biases toward a lower-than-actual estimate of the prevalence of unruptured intracranial aneurysms. These included the review of radiographic reports rather than the radiographs themselves, the inherent sensitivity of angiography, and the lack of complete workups for the $47 \%$ of patients who did not undergo posterior circulation angiography, and the $27 \%$ of patients in whom only unilateral carotid angiography was performed. Given the presence of such significant bias, the authors performed their calculations of rupture incidence based on a prevalence of $1.3 \%$, double the prevalence found in their review. Despite this inflation of prevalence, the risk of bleeding for unruptured intracranial aneurysms was only $0.9 \%$ per year. Unfortunately, calculations made with arbitrary adjustment factors lack statistical validity. Nonetheless, the results of Winn and colleagues, based on an extremely large single-institution data set, provide starkly contrasting estimates compared with the ISUIA results and, as such, are worthy of consideration for the physician hoping to assess the true risks associated with unruptured intracranial aneurysms.

\section{Further Considerations}

There are a number of additional factors to be considered. For instance, many studies break aneurysms into size categories ( such as $<10 \mathrm{~mm}$ or $>10 \mathrm{~mm}$ ). It is highly unlikely there is any "magic size limit" that will result in drastically different rupture rates once crossed. Rather, these are arbitrary boundaries set up by the investigators for ease of data collection, presentation, and comparison. In actuality, the risk of bleeding for unruptured intracranial aneurysms reflects a continuum of increasing likelihood with greater aneurysm size. Additionally, it is important to realize that these entities are not static, but have been demonstrated to undergo growth in a number of studies. ${ }^{2,8,11,21}$ For instance, Juvela, et al., ${ }^{11}$ found that in 31 $(36 \%)$ of 87 patients the size of conservatively managed aneurysms grew at least $3 \mathrm{~mm}$ over a mean follow-up period of 18.9 years. The relative effect of aneurysm growth on our ability to estimate hemorrhage risk is unknown, but it would be illogical to assume that an 8-mm aneurysm will always stay $8 \mathrm{~mm}$ and never become an $11-\mathrm{mm}$ aneurysm, and thereby acquire an increased rupture risk.

Second, any estimation of rupture risk must take into account the aneurysm location. For instance, the results of the ISUIA demonstrate that aneurysms of the PCoA and posterior circulation display a much higher risk of rupture ( 2-7 times higher) than those of the middle cerebral ar- 
tery and ICA. A survey of the literature indicates that posterior circulation, PCoA, and anterior communicating artery aneurysms carry the highest risk of $\mathrm{SAH}$, whereas aneurysms of the cavernous ICA carry an extremely low rupture risk. $1,2,7,24$

Last, it has been our experience that there is a very real phenomenon of excessive psychological stress in patients harboring an unruptured intracranial aneurysm. Even when patients fall into a subgroup of minimal or unknown treatment benefit and are appropriately counseled, they often insist on treatment. Although rigorous studies of this issue have yet to be performed, it appears that the psychological stress associated with harboring an unruptured intracranial aneurysm is great enough cause a patient to forgo the conservative management recommended in the literature for the perceived peace of mind in knowing that a treatment has been exercised. ${ }^{17}$ We would like to stress that our policy is universally to recommend management according to our best interpretation of the literature, but there is a subset of patients who insist on an intervention regardless of the literature-based rates. These patients may be borderline candidates to begin with, and such strong feelings on their part may swing the risk/benefit analysis toward performing an intervention.

\section{CONCLUSIONS}

Despite the variability in recent literature, we believe that it is still possible to make reasonably informed decisions concerning the bleeding risk of unruptured intracranial aneurysms. Before outlining these conclusions we wish to stress that continued investigation is, without a doubt, necessary. It is our hope that the current questions concerning the natural history of these lesions may eventually be answered through high-quality prospective observational studies and perhaps even a randomized trial. For now we feel confident making the following recommendations.

The natural history of a given unruptured intracranial aneurysm should be individually assessed for each case, because there are a number of factors that alter predicted rupture rates. For instance, family history, smoking, excessive alcohol consumption, female sex, previous aneurysmal SAH, presence of symptoms attributable to the lesion, aneurysm location, and lesion size have all been demonstrated to predict a worse natural history. Therefore, all of these factors must be taken into account when evaluating an unruptured intracranial aneurysm. In addition, any risk/benefit analysis of proceeding with intervention must take into account the patient's life expectancy and medical comorbidities. Despite these important concerns, it is helpful to have a general, nonspecific algorithm for predicting rupture risk that may then be adjusted appropriately depending on the aforementioned risk factors.

Of the two ISUIAs, the prospective portion most likely provides the best estimate of the natural history of unruptured intracranial aneurysms. Despite this, the extreme selection bias and questionable study design (that is, exclusion of crossover patients) make it highly likely that this paper underestimates the risk of bleeding in unruptured intracranial aneurysms. The paper by Juvela, et al., is the best designed and executed investigation, but due to its observational nature, it is also subject to significant selection bias, because it has been suggested that the Finnish population has a higher incidence of aneurysmal SAH than the international community. The metaanalysis published by Rinkel, et al., minimizes the biases of individual studies through aggregate analysis and evaluates the largest number of patients. As a result, it provides a very reasonable estimate of rupture risk. Further support for their results is afforded by their close agreement with those of Juvela, et al. Last, Winn, et al., have provided a thorough single-center evaluation of prevalence that likely underestimates the true rate of unruptured aneurysm prevalence. Despite this, even when reasonable corrections are made (such as the doubling of their determined prevalence rate) their results are more in line with those of Rinkel, et al., and Juvela, et al., than those reported in the retrospective ISUIA.

Taking the results of all these studies together, we believe that a good general estimate for the yearly risk of SAH for an unruptured intracranial aneurysm is approximately $1 \%$ for lesions 7 to $10 \mathrm{~mm}$ in diameter. The risk of rupture grows as aneurysm size increases, and likewise diminishes as aneurysm size decreases. This general rule serves as a reasonable interpretation of the results reported in the current body of literature.

\section{References}

1. Asari S, Ohmoto T: Natural history and risk factors of unruptured cerebral aneurysms. Clin Neurol Neurosurg 95: 205-214, 1993

2. Benson K, Hartz AJ: A comparison of observational studies and randomized, controlled trials. N Engl J Med 342:1878-1886, 2000

3. Concato J, Shah N, Horwitz RI: Randomized, controlled trials, observational studies, and the hierarchy of research designs. $\mathbf{N}$ Engl J Med 342:1887-1892, 2000

4. Dell S: Asymptomatic cerebral aneurysm: assessment of its risk of rupture. Neurosurgery 10:162-166, 1982

5. Dippel DW, Habbema JD: Natural history of unruptured aneurysms. J Neurosurg 80:772-774, 1994

6. Hademenos GJ, Massoud TF, Turjman F, et al: Anatomical and morphological factors correlating with rupture of intracranial aneurysms in patients referred for endovascular treatment. Neuroradiology 40:755-760, 1998

7. The International Study of Unruptured Intracranial Aneurysms Investigators: Unruptured intracranial aneurysms - risk of rupture and risks of surgical intervention. N Engl J Med 339: 1725-1733, 1998

8. Juvela S: Natural history of unruptured intracranial aneurysms: risks for aneurysm formation, growth, and rupture. Acta Neurochir Suppl 82:27-30, 2002

9. Juvela S, Porras M, Heiskanen O: Natural history of unruptured intracranial aneurysms: a long-term follow-up study. J Neurosurg 79:174-182, 1993

10. Juvela S, Porras M, Poussa K: Natural history of unruptured intracranial aneurysms: probability of and risk factors for aneurysm rupture. J Neurosurg 93:379-387, 2000

11. Juvela S, Poussa K, Porras M: Factors affecting formation and growth of intracranial aneurysms: a long-term follow-up study. Stroke 32:485-491, 2001

12. King JT Jr, Glick HA, Mason TJ, et al: Elective surgery for asymptomatic, unruptured, intracranial aneurysms: a cost-effectiveness analysis. J Neurosurg 83:403-412, 1995

13. Leblanc R, Worsley KJ: Surgery of unruptured, asymptomatic aneurysms: a decision analysis. Can J Neurol Sci 22:30-35, 1995 
14. Linn FH, Rinkel GJ, Algra A, et al: Incidence of subarachnoid hemorrhage: role of region, year, and rate of computed tomography: a meta-analysis. Stroke 27:625-629, 1996

15. Nakagawa T, Hashi K: The incidence and treatment of asymptomatic, unruptured cerebral aneurysms. J Neurosurg 80: 217-223, 1994

16. Ohaegbulam SC: Geographical neurosurgery. Neurol Res 21: 161-170, 1999

17. Otawara Y, Ogasawara K, Kubo Y, et al: Anxiety before and after surgical repair in patients with asymptomatic unruptured intracranial aneurysm. Surg Neurol 62:28-31, 2004

18. Rinkel GJ, Djibuti M, Algra A, et al: Prevalence and risk of rupture of intracranial aneurysms: a systematic review. Stroke 29: 251-256, 1998

19. Samson DS, Hodosh RM, Clark WK: Surgical management of unruptured asymptomatic aneurysms. J Neurosurg 46: 731-734, 1977

20. Solomon RA, Fink ME, Pile-Spellman J: Surgical management of unruptured intracranial aneurysms. J Neurosurg 80: 440-446, 1994

21. Suga M, Yamamoto Y, Sunami N, et al: [Growth of asymptomatic unruptured aneurysms in follow-up study: report of three cases.] No Shinkei Geka 31:303-308, 2003 (Jpn)
22. Tomasello F, D’Avella D, Salpietro FM, et al: Asymptomatic aneurysms. Literature meta-analysis and indications for treatment. J Neurosurg Sci 42 (Suppl 1):47-51, 1998

23. Ujiie H, Sato K, Onda H, et al: Clinical analysis of incidentally discovered unruptured aneurysms. Stroke 24:1850-1856, 1993

24. Wiebers DO, Whisnant JP, Huston J III, et al: Unruptured intracranial aneurysms: natural history, clinical outcome, and risks of surgical and endovascular treatment. Lancet 362: 103-110, 2003

25. Winn HR, Jane JA Sr, Taylor J, et al: Prevalence of asymptomatic incidental aneurysms: review of 4568 arteriograms. J Neurosurg 96:43-49, 2002

26. Yasui N, Suzuki A, Nishimura H, et al: Long-term follow-up study of unruptured intracranial aneurysms. Neurosurgery 40: 1155-1160, 1997

Manuscript received October 7, 2004.

Accepted in final form October 20, 2004.

Address reprint requests to: E. Sander Connolly Jr., M.D., Columbia University College of Physicians and Surgeons, 710 West 168th Street, Room 435, New York, NewYork 10032. email: esc5@columbia.edu. 